Cinémas

Revue d'études cinématographiques

Journal of Film Studies

\title{
Entre présent et possible : la difficile recomposition du paysage de la banlieue dans le film de Mathieu Kassovitz, La Haine
}

\section{Jean Mottet}

Volume 12, numéro 1, automne 2001

Le Paysage au cinéma

URI : https://id.erudit.org/iderudit/024868ar

DOI : https://doi.org/10.7202/024868ar

Aller au sommaire du numéro

Éditeur(s)

Cinémas

ISSN

1181-6945 (imprimé)

1705-6500 (numérique)

Découvrir la revue

Citer cet article

Mottet, J. (2001). Entre présent et possible : la difficile recomposition du paysage de la banlieue dans le film de Mathieu Kassovitz, La Haine. Cinémas, 12(1), 71-86. https://doi.org/10.7202/024868ar
Résumé de l'article

Depuis ses débuts, l'oeuvre filmique est saisie par la ville. Que se passe-t-il au cinéma quand, dans le réel, le paysage urbain se désagrège, notamment dans le cas des banlieues? Pour comprendre cette nouvelle « manière d'être à l'espace », nous examinerons d'abord quelques aspects des cheminements des corps dans le film La Haine, de Mathieu Kassovitz. Il faut commencer à réenvisager les nouveaux paysages urbains au plus près de leurs fréquentations ordinaires, quotidiennes, là où sautent les maillons. Ces déambulations sont aussi à la recherche de nouvelles connexions avec le paysage. En insérant la rupture dans la manière d'occuper l'espace, le film, implicitement, suggère d'autres liaisons imaginaires. Ainsi, porté par le paysage sonore, le film engendre parfois de brefs échanges entre le geste et la cité. 


\section{Entre présent et possible: la difficile recomposition du paysage de la banlieue dans le film de Mathieu Kassovitz, La Haine}

\section{Jean Mottet}

\section{RÉSUMÉ}

Depuis ses débuts, l'œuvre filmique est saisie par la ville. Que se passe-t-il au cinéma quand, dans le réel, le paysage urbain se désagrège, notamment dans le cas des banlieues? Pour comprendre cette nouvelle «manière d'être à l'espace", nous examinerons d'abord quelques aspects des cheminements des corps dans le film $\mathrm{La}$ Haine, de Mathieu Kassovitz. Il faut commencer à réenvisager les nouveaux paysages urbains au plus près de leurs fréquentations ordinaires, quotidiennes, là où sautent les maillons. Ces déambulations sont aussi à la recherche de nouvelles connexions avec le paysage. En insérant la rupture dans la manière d'occuper l'espace, le film, implicitement, suggère d'autres liaisons imaginaires. Ainsi, porté par le paysage sonore, le film engendre parfois de brefs échanges entre le geste et la cité.

\section{ABSTRACT}

Film, from its very beginnings, has been drawn to the city. What happens to the cinema when real urban landscapes disintegrate into suburbs? As a way of understanding this new way of "being in space," the author first discusses different aspects of the body's trajectories in Mathieu Kassovitz's film La Haine. We must 
find a new way of looking at new urban landscapes, in keeping with their habitual and day-to-day use, in the space where the links of the chain are broken. These suburban trajectories are also searching for new connections with the landscape. The film implicitly suggests new, imaginary liaisons in the way it introduces rupture into the manner of occupying space. In this way, carried along by the aural landscape, the film at times gives rise to brief exchanges between the gesture and the city.

Dans l'univers des représentations filmiques, la place occupée aujourd'hui en France par la banlieue (celle de Paris, essentiellement) s'inscrit dans une histoire déjà longue qui, sur bien des

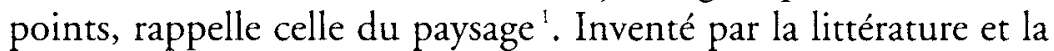
peinture dans la seconde moitié du XIX siècle, le paysage de la banlieue semble ensuite exiger sans cesse de nouvelles formes d'appréciation. C'est ainsi qu'il a été constamment accompagné dans son évolution, dans ses mutations, par un ensemble de représentations (photographie, cinéma, bande dessinée, chanson, etc.) qui, à leurs différentes façons, se sont emparées des dispositions variées de ses espaces ${ }^{2}$. Pourquoi ce paysage ordinaire, banal, suscite-t-il autant de représentations, donnant parfois le sentiment de réclamer des modes d'appropriation plus adaptés? Quelle est la place du cinéma dans cette histoire?

"Il est certain, écrit Éric Rohmer (1985), que la banlieue offre une matière de choix au cinéaste. D'abord parce que des millions de gens y habitent, ensuite parce que c'est un décor plus neuf, plus varié que Paris ${ }^{3}$." Je ne pense pas que l'on puisse réduire le paysage de la banlieue à un "décor", à un simple fond pour les corps et les visages. Les films de Rohmer, par l'introduction répétée du réel dans l'œuvre, en font d'ailleurs euxmêmes la démonstration. Dans les années trente, Marcel Carné, quant à lui, envisageait le retour à la banlieue comme un retour à "l'esprit initial" du cinéma et n'hésitait pas à utiliser le mot paysage pour qualifier la périphérie (Carné, 1987). Qu'en est-il aujourd'hui?

Apparemment, si l'on en croit la plupart des analyses urbaines, nous serions devant un échec des nouveaux ensembles créés dans les périphéries. Les bonnes questions ont-elles été posées? 
Car nous sommes, depuis une dizaine d'années, à la fois dans une période d'évolution rapide du paysage (réel) des banlieues et à un moment de basculement des représentations. Or habiter ces nouveaux espaces, demeurer dans ces nouveaux lieux, éventuellement les transformer en paysage, cela implique de profonds changements dans les pratiques d'appropriation paysagère, comme dans les modes d'approche de ces pratiques. Comment l'idée de banlieue s'est-elle modifiée? Comment des images positives, des paysages ont-ils pu naitre dans cet univers si décrié pour ses conditions de vie? Dans un mouvement comparable au processus d' "artialisation" défini par Alain Roger pour le paysage ${ }^{4}$, je pense que le cinéma d'aujourd'hui participe activement, sur le plan imaginaire, à la constitution de configurations spatiales fondatrices de ces nouveaux paysages de la banlieue. L'itinéraire proposé par le film est cependant déroutant, car les représentations proposées ne s'inscrivent pas dans les termes habituels des approches urbaines ou paysagères. En fait, au travers d'une autre saisie de l'espace, le cinéma nous invite à penser autrement les particularités de ces paysages ordinaires. D'une certaine façon, dans ce goût pour les paysages déshérités, le cinéma français renouerait avec une très ancienne disposition ${ }^{5}$. En se référant à l'une des propositions faites par Antonio Costa, dans son intéressante classification des usages et des fonctions du paysage au cinéma, on peut même voir dans cette permanence de l'attachement au paysage de la banlieue l'indication que ce dernier est effectivement une "composante des caractères originaux du cinéma français" (1999, p. 173).

Au risque de réduire l'analyse d'un phénomène français de société à un type d'espace, nous proposerons ici d'interroger "la nouvelle manière d'être à l'espace " qui se dégage du film $L a$ Haine de Mathieu Kassovitz. Car comprendre ce qu'habiter les cités de la banlieue veut dire aujourd'hui implique une redéfinition de notre rapport à l'espace, aux lieux, et au paysage. Il y a toujours des lieux, mais aussi d'autres espaces que des lieux, sans pour autant nécessairement les transformer en "non-lieux ${ }^{6}$ ». D’où ma préférence, ici, pour la notion de «hors-lieu», résultat de la dislocation des lieux. Allant dans le sens de Benoît Goetz, qui note que "le "dis-" de la dislocation n'est pas d'abord, en 
rien, destructeur» $\left(1997\right.$, p. $\left.100^{7}\right)$, je dirais que le «hors-» du hors-lieu n'a pas nécessairement une connotation négative. C'est un autre mode de relation qui se cherche. Quel rôle le paysage joue-t-il dans la détermination des nouveaux sites? Les objets architecturaux induisent-ils une habitabilité? Comment la périphérie se rattache-t-elle, s'articule-t-elle aux autres lieux (à la campagne, à Paris) ?

\section{La violence d'être hors lieu}

"Il m'a enfermé dehors", s'exclame Saïd, l'un des trois personnages principaux du film, quand un taxi parisien refuse de le prendre. Cette phrase résume assez bien, me semble-t-il, la situation spatiale d'exclusion des trois jeunes héros du film, assignés à résidence en quelque sorte dans la cité de Chanteloup-les-Vignes. Comme on le sait, les habitants de ce type de cité ne choisissent pas vraiment le lieu de leur résidence. Et l'expression de Said dans le film ferait ainsi écho à une série d'autres, par exemple celles que rapportent les enquêteurs sur le terrain. "Ils" nous ont mis "là ", affirment souvent les habitants interviewés. "Là ", c'està-dire au "ban du lieu ", selon l'expression de Daniel Sibony dans son admirable analyse de la violence des jeunes. "Là ", quelque part entre la Ville et la Nature, une zone indéterminée en quelque sorte. «Être enfermé dehors quand dedans il n'y a rien, le rien où marinent ceux qui sont dedans" (Sibony, 1998, p. 104). Mais qu'est-ce qui se passe quand on est enfermé dehors?

Dans le domaine des études cinématographiques, on connaît l'importance des relations entre les réalisateurs et le jeu des acteurs. On commence à peine à reconnaître l'importance des occupations par les corps des lieux, des espaces, des paysages dans une redécouverte de l'une des qualités du cinéma qui est, en effet, cette capacité à enregistrer les corps en mouvement, leur façon de marcher, par exemple. C'est ainsi que Michel Chion, à juste titre, a pu parler de "metteurs en scènes de la marche» (1995, p. 37-51). Dans cette perspective, relativement nouvelle, de confrontation des corps avec leur environnement réel, il y a, me semble-t-il, des occupations quotidiennes de l'espace, banales, apparemment insignifiantes, mais où le geste anodin, contre la dislocation, fait naître des lieux nouveaux, des 
mondes nouveaux, qui se donnent à voir au cinéma. Je pense que c'est le cas de certains "banlieues-films" ", notamment $\mathrm{La}$ Haine, où ce qui fait la cité doit aussi être recherché dans les représentations de ces pratiques banales, et où ce qui fait le paysage urbain relève d'un regard qu'on peut dire rapproché. Ce regard rappelle à certains égards le " regard de près " qui est, selon Daniel Arasse, caractéristique de "l'oscillation essentielle du regard en peinture, née à propos de la peinture de paysage " (1996, p. 252). Pour cet historien de l'art, cette peinture «aurait eu le privilège d'une dislocation autorisée du regard». Bien que l'analyse d'Arasse porte sur le spectateur de tableau, où un autre regard, "de loin », global, continue d'organiser la scène, la similitude avec le regard qui fait le paysage est troublante, d'autant plus qu'à ses yeux, la "dislocation" a également pour conséquence ce qu'il appelle une "dislocalisation», au cours de laquelle, perdant le regard que lui donne le point de vue, le corps se retrouve dans le paysage, dans le tableau et en parcourt le "caractère local" (p. 251).

Vinz, Saïd, Hubert ne tiennent pas en place, bougent sans cesse. Comment qualifier ces mouvements? Ces cheminements ont-ils un sens? Que nous apprennent-ils sur l'espace de la cité? Sont-ils de simples répétitions, des ressassements? Ou bien y at-il une "rhétorique des cheminements", selon l'expression de Michel de Certeau ${ }^{9}$ ? Serait-ce une rhétorique elle-même organisée par la cité, ses contraintes architecturales ${ }^{10}$ ?

On verra ici que ce ne sont pas uniquement les contraintes topographiques locales de la cité qui conditionnent les déplacements des protagonistes. Pour plusieurs raisons. Tout d'abord, parce que les "objets architecturaux" de la cité, les scénographies de Chanteloup-les-Vignes, lieu de tournage, mais aussi lieu diégétique réel, ne sont pas des lieux organisateurs de sens (comme on peut en trouver chez Rohmer, par exemple dans un film comme L'Ami de mon amie). Non seulement la cité de Chanteloup-les-Vignes n'est pas belle (perception esthétique), mais elle n'est pas lisible, c'est-à-dire, selon Kevin Lynch (1999) à qui j'emprunte ce concept de lisibilité, que les repères spatiaux ne s'organisent pas en un tout cohérent. Il faut bien voir que pour Lynch cette lisibilité peut s'organiser selon des formes 
simples (limites, chemins, nœuds, repères). L'hégémonie et l'esthétisme du Beau ont fait méconnaître, nous suggère-t-il, le fait que le milieu construit a pour qualité spécifique d'être signifiant. Pour le comprendre, il faut partir du bas, nous dit Michel de Certeau, pour qui "l'histoire commence au ras du sol, avec des pas" (1980, p. 179). On le voit, l'apparente dislocation n'implique pas nécessairement l'absence de cohérence.

Les jeunes ne cessent de bouger, le plus souvent à pied, et leur marche elle-même est déjà, en soi, l'indication d'un manque de lieu. Mais nous ne sommes pas dans un film de Pasolini, où marcher dans la banlieue (dans les borgate de Rome) devient le signe d'une "non-participation " à la vie normale de la petite bourgeoisie, où marcher devient un moyen de s'opposer, par l'expression d'une sorte de narrativité contestatrice assortie d'un engagement politique (à gauche). Que ce soit sur la route (asphaltée) ou sur les sentiers (à peine tracés) des terrains vagues, les déambulations des personnages pasoliniens fondent un rapport au monde, au paysage. Dans des films comme Mamma Roma ou Accatone, la route, le chemin, les sentiers ne se réduisent pas à des motifs ou à des thèmes. Par les incessants mouvements d'approche et d'éloignement que ceux-ci suscitent, ils maintiennent une connexion avec le monde, offrent des prises dans le paysage. Je pense par exemple ici à l'étonnant parcours amoureux d'Ettore et de Bruna sur les sentiers poussiéreux, à peine tracés, des terrains vagues dans Mamma Roma.

Avec le film de Kassovitz (mais également avec la plupart des "banlieues-films» des années quatre-vingt-dix), nous sommes plutôt dans l' "infrapolitique", dans une contestation plus radicale, sans espoir aucun. La Haine est un cri de désespoir contre une injustice définitive, fondamentale. Et la marche des personnages, leur extrême mobilité ne sont pas sans rapport avec ce cri, cette totale désespérance. Mais voyons cela avec plus de précision, au plan de l'espace.

Le trio parcourt des lieux, traverse des cours, emprunte des passages, des aires de jeu, se réfugie dans des caves ou alors pique-nique sur les toits (après le parcours sol terrain, le parcours aérien, que l'on retrouvera dans les déplacements à Paris). Ce qui me frappe, c'est l'absence de direction: le trio donne 
l'impression de tourner en rond. Sans être franchement hostile (Paris sera plus risqué), les espaces parcourus dans la cité restent disons incohérents, stagnants, illisibles.

Autrement dit, il y a de l'espace, de l'espace rendu sensible, notamment par les incessants déplacements (des personnages et de la caméra), mais pas vraiment de lieu. Selon certains ethnologues, un lieu est un espace pourvu de sens, un sens qui est créé entre autres par la manière dont on occupe cet espace, la façon dont on l'habite, ou encore par une architecture qui précise l'espace, le transforme en lieu. Ce n'est ni le lieu ni le non-lieu, au sens de Marc Augé. Ce serait plutôt l'espace, c'est-à-dire le «lieu pratiqué» au sens de Michel de Certeau, selon lequel «le lieu est une configuration instantanée de positions [qui] implique une indication de stabilité... L'espace est un croisement de mobiles...» (1980, p. 308).

De ce point de vue, l'absence de route et de chemin, dans une cité pourtant parcourue sans relâche, me paraît révélatrice d'une nouvelle manière d'être. La comparaison avec Pasolini est ici encore éclairante. Dans les borgate, loin des espaces construits du centre de Rome dominé par les monuments, les personnages pasoliniens partent à la recherche d'un pays plus libre, plus sauvage, notamment en empruntant des routes, des chemins, des sentiers, qui, dans les marges, s'essaient à modifier le rapport aux lieux. Dans La Haine, au contraire, les jeunes semblent avoir renoncé à établir le contact avec un monde réduit à un objet extérieur, inaccessible, hostile. Emportés par un rythme infernal, les corps ne se laissent jamais aller aux choses. Et sans ces sensations que le corps recueille au contact du chemin, pas de plaisir, pas de jubilation; les corps, ici, semblent avoir perdu toute capacité d'enthousiasme. Les déplacements, presque toujours latéraux, sont rapides, accompagnés par des travellings en steadicam, pour mieux accentuer l'aspect fluide, coulé des mouvements, notamment dans les passages entre immeubles, dans le labyrinthe des caves. À cette vitesse de déplacement, à l'extrême mobilité, succèdent l'arrêt, la stagnation des corps. Vinz et Hubert sont en effet souvent montrés en position couchée, sans pour autant que l'on puisse parler de véritable posture ${ }^{11}$. Plutôt que des postures, on a ici affaire à des allures (style, vitesse de 
mouvement), des pauses plutôt que des poses. Dans leurs arrêts comme dans leurs mouvements, les corps des jeunes semblent vouloir éviter tout ce qui risquerait de faire naître un rapport au monde, par le biais d'une attitude de contemplation par exemple. Aucun désir non plus ne semble motiver leurs déplacements, sans but précis; ce qu'ils recherchent, c'est plutôt la possibilité d'un désir.

Selon des études sociologiques récentes, cette rapidité des déplacements des jeunes, constatée sur le terrain des cités, correspondrait à une modification récente de leurs comportements: "Les jeunes des banlieues se déplacent de plus en plus vite, et de manière de moins en moins visible" (Bordet, 1998, p. 119128).

Dans le film, effectivement, les mouvements du trio me semblent directement affectés par ces nouveaux rythmes temporels de la ville. Rythmes qui ne sont pas sans rapport, par ailleurs, avec la course vers l'apocalypse qui, dans La Haine, scande le film, mesurée par le tic-tac infernal accompagnant les images, dans une accélération qui transforme l'évolution en explosion.

Dans le cadre de cette nouvelle temporalité de l'urbain resteraient donc des déplacements nerveux, d'une nervosité qui déplacerait, disperserait les anciens trajets (par exemple, le nonrespect des sentiers prévus entre les pelouses des cités). Faut-il voir là des tentatives pour s'approprier un lieu? Terme vague, mais qui correspond assez bien à cet ensemble de gestes et de mouvements qui vise à créer un "sentiment de chez soi ", où l'on est bien, où l'on n'est pas rejeté.

Les anciens trajets sont remplacés par de brusques embardées des corps, suivies de replis sur soi (Hubert). Bref, des mouvements qui exprimeraient la vie dans le présent, l'immédiat, sans perspective, sans anticipation, sans choix, sans futur. Dans une réflexion sur les rapports lieu/hors-lieu sur le plan temporel perspective intéressante dans le cas de La Haine, mais que nous ne pouvons malheureusement développer dans le cadre de cet article - ces notions deviennent des états momentanés du site. Le hors-lieu ne serait alors qu'un lieu qui change trop vite ou qui ne change pas du tout, sans passé, sans avenir, à l'image des trois personnages du film. Le lieu semble avoir perdu la capacité 
d'intégrer l'événement dans le temps. N'est-ce pas là d'où origine le sens de "demeurer ", étymologiquement dérivé de la notion de durée (du latin mora)?

Ensuite, ce qui structure la perception de l'espace, ce n'est pas seulement ce que le sujet a matériellement sous les yeux. Les espaces présents autour du lieu de vie jouent un rôle important. Avec le "banlieue-film" à la manière de Kassovitz, la proximité, réelle et imaginaire, d'autres lieux (Paris, notamment) nous invite à mettre en œuvre notre aptitude à l'imagination globale. Comme le film le montre bien, par exemple au travers des quelques instants que dure le voyage à Paris, la capitale est à la fois proche $^{12}$ et inaccessible (les trois jeunes y seront plus malheureux encore). Et visiblement l'imaginaire ne suffit pas à en faire la synthèse.

Mais Paris n’est pas le seul lieu extérieur évoqué. Pendant le voyage, Hubert aperçoit pour la première fois, depuis un train en mouvement, une affiche représentant le globe terrestre accompagné du slogan "Le monde est à vous». La Terre est le lieu des lieux, le lieu de leurs attentes, de nos attentes, de leurs impossibilités surtout, car la mort règne et se superpose au globe terrestre, sous la forme d'un cocktail Molotov.

La perte de signification de l'espace de la cité, son instabilité seraient aussi dues à la difficulté à relier le particulier au général, le proche et le lointain à la proximité, la richesse potentielle (et illusoire) d'autres mondes, voire du monde ${ }^{13}$. Dans son invitation à poursuivre l'épopée humaine, à ouvrir un nouvel espace de rêve, le slogan publicitaire "Le Monde est à vous" de l'affiche pointe le lieu qui manque et souligne ironiquement le trouble dans lequel nous rencontrons notre monde aujourd'hui, la difficulté de l'humanité à devenir Humanité. L'image du globe terrestre, reproduite sur l'affiche, renvoie en fait à deux aspects de notre planète: d'une part, la Terre entendue disons comme élément poétique, évoquée depuis toujours, et d'autre part le globe terrestre envisagé comme objet, construit par la technique (la photographie de l'affiche est une reproduction d'une vue de la Terre prise pendant le premier vol vers la lune en 1969). Comment se repérer dans le monde global qui s'impose quand, parmi les changements, l'un des plus importants concerne préci- 
sément notre manière d'habiter le monde, de le parcourir ${ }^{14}$. À cet égard, il n'est pas indifférent de noter que la première apparition de l'image du globe terrestre dans le film a lieu à l'occasion du voyage des trois jeunes à Paris, un voyage rapide, nous l'avons vu, qui tend à éliminer le temps. Reste un pur trajet où s'effacent le sujet et l'objet. Hubert regarde-t-il ce qu'il voit par la fenêtre du train? D'où l'attitude détachée des jeunes par rapport à la cité, à l'environnement proche, et l'échec de la sortie à Paris. Car pour rencontrer le monde, le contempler (une forme de rencontre), il faut peut-être, d'abord, habiter quelque part. Du moins c'est-ce ce que nous disent des philosophes comme Levinas ou Heidegger. "Le sujet contemplant le monde suppose l'événement de la demeure", affirme Lévinas (1961, p. 164). Autrement dit, celui qui est chassé de la maison est aussi chassé du monde.

\section{Les relations dehors/dedans: l'affaiblissement des limites}

Le rempart, la rue, la place forment des limites, nous disent les urbanistes, tout comme la porte, le toit, le mur sont les limites d'une maison. Ces limites facilitent les repères, donnent à voir, à comprendre, à vivre des lieux. Qu'en est-il des espaces représentés dans La Haine, à un moment où l'on considère généralement que les limites de la ville se perdent dans "l'urbain généralisé"?

Si l'on examine attentivement les cheminements du trio dans la cité, plus exactement leurs entrées et leurs sorties, leurs passages de l'intérieur à l'extérieur (relations essentielles à la définition de l'identité d'un lieu, nous disent les architectes, les anthropologues), on note une absence assez remarquable de ce que j'ai appelé ailleurs "espaces de transition ${ }^{15}$ ". Par "espace de transition", j'entends, par exemple, le palier, l'escalier, l'entrée d'immeuble, le seuil, des objets architecturaux articulant le "dehors" et le "dedans", le privé et le voisinage. Dans La Haine, Kassovitz choisit de ne pas nous montrer ces traversées d'espaces proches, le moment où l'on s'approche de chez soi, l'arrivée dans le "très proche", le franchissement du seuil, cette "promesse d'intérieur" selon la belle formule de Gaston Bachelard. Sans ces mouvements qui nous accommodent au voisinage, c'est tout le rapport à la cité qui se désagrège, se disloque. "Partout, les maillons sau- 
tent, quand, peut-être, il suffit d'un manque pour que la phrase terrestre n'ait plus de sens ", écrit Yves Bonnefoy (1977, p. 132).

En éliminant ces traversées d'espaces proches, c'est l'espace du chez soi que l'on supprime. Comme le remarque J.-F. Lyotard dans un chapitre de son beau livre L'Inhumain, intitulé "Domus et la mégapole»: "l'espace domestique s'enlace des circonvolutions, des allers et venues de la conservation" (1998, p. 204). Kassovitz montre des mouvements (marcher, traverser, prendre le train, monter, descendre...), mais les fragments ne se rejoignent jamais, ne se localisent pas. Ce que nous dit le film de Kassovitz, à sa façon, par cette réorganisation des lieux, de l'espace, c'est que la maison est devenue impossible. "Nous nous éveillons et nous ne sommes pas heureux. Pas question de refaire une vraie jeune maison» (Lyotard, 1998, p. 209). La figure récurrente de l'homme fatigué, couché dans sa chambre (c'est le cas de Vinz et d'Hubert à plusieurs reprises), que Kassovitz emprunte au Scorsese de Mean Streets et de Taxi Driver, exprime bien ce malaise au réveil.

Tous les "banlieues-films" ne sont pas autant dépourvus de convivialité que La Haine. Dans un film intéressant de Karim Dridi, Bye Bye (1951), dont l'action se situe, il est vrai, dans une banlieue de Marseille, la beauté des images et la lumière font vivre sensuellement, physiquement le "très proche", le voisinage de l'appartement, l'escalier, le seuil. De ce point de vue, un film comme Mon Oncle (1958) de Jacques Tati rassemble de manière emblématique, dans sa description du logement de M. Hulot, tous les éléments d'une bonne communication dedans/dehors ${ }^{16}$.

Par ailleurs, cette perte du lieu est aussi liée à l'émergence des systèmes modernes de communication qui, en modifiant les relations au clehors, font disparaître ou, du moins, contribuent à affaiblir la spécificité des lieux. Le dehors ne communique plus avec le dedans, si ce n'est par les bruits dont on tente de se protéger en se retranchant dans une boîte, avec, au centre, le seul canal qui relie au dehors: la télévision, allumée vingtquatre heures sur vingt-quatre, diffusant des images qui racontent le dehors. Plusieurs questions se posent. Quelle vision paysagère peut avoir une collectivité qui n'a devant les yeux que des images venues d'ailleurs? Quels sont les lieux contemporains des nouvelles technologies? 


\section{Du paysage sonore à la tentation de l'illimité}

«Dans ce film, on entend le son des banlieues», remarque Thierry Jousse dans les Cahiers du cinéma. De quel son s'agit-il? Le son de la voix, d'abord. Ce qui donne sa force au film, c'est son langage, sa langue plutôt que le récit, le scénario ou même la musique, dont nous allons parler. Si l'on marche pas mal, on parle tout le temps, avec une avalanche de mots, d'expressions, voire de vociférations, d'interjections. Le tout dans un rythme assez infernal, qui donne sa pulsation au film. "Ça parle, ça veut dire, mais ça ne peut pas. Ça frôle l'indicible», écrit Daniel Sibony (1998, p. 104).

Mais ce qui m'intéresse ici, c'est le son en situation, dans l'espace de la cité. Car les sons sont souvent amplifiés par des effets de réverbération sur les immeubles, dans les cages d'escalier ${ }^{17}$. En intérieur comme en extérieur, les lieux sont porteurs de sons. Parfois, dans un même lieu, le paysage sonore se modifie à la suite des activités des jeunes. Si bien que l'on peut avancer l'hypothèse d'une appropriation de l'espace par le son. " Ça manque d'ambiance", disent souvent les jeunes des cités! La très belle séquence où le Dee Jay, de l'intérieur, lance sa musique vers la cité est particulièrement intéressante de ce point de vue.

Au départ, il y a ce plan du jeune Dee Jay, de dos, face à la fenêtre, face à la cité (que l'on voit), qui se concentre pour diffuser sa musique vers la cité. Une communication s'établit entre "dedans" et "dehors", signifiée par la forte présence de la fenêtre qui, exceptionnellement, ouvre sur la cité. Bien qu'il n'y ait pas de public pour l'écouter, on est pas loin ici du jeu en public, c'est-à-dire d'une performance où la musique a une forte relation à l'espace. Aidée, portée en quelque sorte par le travelling aérien, la musique est produite pour se répandre vers l'extérieur, pour créer une ambiance sonore, voire imprimer sur le territoire de la cité une "marque jeune», par le son. De ce point de vue, on peut distinguer deux moments contradictoires et simultanés: une échappée, une fuite (au-dessus des toits, vers l'horizon) et une sorte de "reterritorialisation" (par les paroles de la chanson de Piaf évoquant la France de toujours).

Du mélange d'une musique d'inspiration américaine et de celle de NTM (groupe de rappeurs français) naît un sentiment 
de révolte, clairement exprimé par la répétition de «Nique la police ${ }^{18} \%$. Un espoir, ensuite, un petit moment d'utopie, c'està-dire de sortie du temps vers un autre temps, avec l'aide d'un voyage par-dessus les toits, vers l'horizon, dans une vision rêvée de la cité. Le rappeur ne se présente-t-il pas souvent comme une sorte de messie avec qui les temps prennent fin et commence le grand jugement. Mais le futur semble ici relié au passé, par le retour d'un air français particulièrement populaire, la chanson de Piaf, comme si le rappeur semblait avoir besoin, pour produire une autre musique, de ce double ancrage dans le passé et dans le territoire.

Ce travail sur les sons et sur la musique accompagne un très beau travelling aérien, qui part de la façade de l'immeuble, vu de l'extérieur, en position frontale, pour survoler ensuite l'aire de jeu, à travers un regard vertical réduisant les jeunes à quelques points abstraits se déplaçant sur la surface bétonnée. Belle illustration, anticipée, de l'affirmation de Vinz qui, sur les toits de Paris la nuit, s'exclamera: "Je me sens comme une petite fourmi perdue dans l'univers intergalactique. » Au moment où il prononce ces mots, la caméra cadre en gros plan l'arrière du crâne de Vinz, qui masque entièrement la vue sur Paris, bloquant toute relation contemplative avec le paysage urbain.

Les choses se passent différemment à Chanteloup-les-Vignes. Le morceau de territoire de la cité, vu d'en haut, sans aucune profondeur, avec une certaine géométrisation des lignes, crée une dimension plus abstraite, loin des repères habituels, offrant ainsi, me semble-t-il, une vision mentale, rêvée du paysage urbain. Quelques très belles images d'arbres évacuent, le temps d'une envolée de la caméra, la laideur de la cité, qui lève le visage de sa surface bétonnée vers le ciel. Profondément transformé par l'association du mouvement de caméra, du point de vue vertical et de la musique, l'espace aménagé de l'aire de jeu nous suggère cette fois un possible imaginable, comme si quelque chose "débutait" (au sens narratif). Peut-être n'est-il pas inutile de remarquer que ce nouveau départ s'amorce à partir de ce qui, dans le paysage urbain du film, ressemble le plus à une place, à un espace en attente de gestes ou de paroles qui se feraient leur chant.

Mais peut-on parler de paysage? Car ce qui définit le regard vertical, comme l'a bien montré Philippe Dubois dans sa belle 
analyse d'une vue Lumière intitulée Panorama pris d'un ballon captif, c'est précisément l'absence d'horizon, absence qui, selon lui, "évacue le paysage de l'image " (1999, p. 30). Pas de paysage sans lointain, sans horizon, nous dit également Michel Collot (1988) qui a écrit un livre passionnant sur les rapports entre horizon et paysage en poésie.

Alors, il faut retrouver l'horizon. Comment comprendre l'échappée par dessus les toits de la cité, à la fin du travelling aérien, l'irruption soudaine d'un horizon, absent pendant tout le film? La présence de l'horizon à l'image est susceptible de nombreuses interprétations. S'agit-il de l'expression d'un espoir ou d'une quête vouée au désespoir? Le désir, ici, ne reconnaît plus de limite: il se porte aux extrêmes, ce qui renforce l'appel du lointain. Mais celui-ci, sans l'aide d'architectures qui auraient pu rétablir une articulation avec le proche (les façades des immeubles HLM, effacées par leur entassement, n'y suffisent pas), n'offre guère d'image rassurante. Après avoir sauté les liaisons entre les fragments d'espaces proches, le film déborde les limites de l'espace. Le franchissement onirique des limites, la rupture des attaches avec la terre exposent au vertige d'un pur imaginaire. Du haut des immeubles, les sons eux-mêmes semblent perdre leur direction. La chute et la mort, dès lors, paraissent inévitables.

\section{Université François-Rabelais (Tours)}

\section{NOTES}

1. Pour ce qui concerne l'invention du paysage à la Renaissance, voir l'ouvrage fondamental d'Alain Roger, Court Traité du paysage (1997). Pour ce qui est du cinéma, comme le remarque très justement José Baldizzone, dans l'excellent numéro des Cabiers de la Cinémathèque consacré à la représentation de la banlieue au cinéma: "Filmer la banlieue en 1984, 1987, 1993, c'est se placer consciemment ou pas dans une constante du cinéma français, dont les formes classiques ont été dessinées dans les années trente par Marcel Carné" (Baidizzone, 1994, p. 108).

2. Pour une étude chronologique de ces représentations, on se reportera à l'excellente analyse de Jacques Van Waerbeke (1991), Images d'espaces de la banlieue de Paris, $X I X$ et XX siècles. Etude de géographie culturelle.

3. Sur le cas particulier du paysage chez Rohmer, voir la belle étude de Philippe Molinier (1999), "Y a-t-il des paysages dans les films d'Éric Rohmer?».

4. "Nous serions devant nos villes et même nos campagnes, dans le même dénuement perceptif (esthétique) qu'un homme du XVII siècle face à la mer et à la montagne. C'est un "affreux pays", qui ne suscite que la répulsion" (Roger, 1997, p. 113). 
5. Voir à ce sujet l'article de J.-P. Le Dantec, "Zones. Les paysages oubliés" (1999, p. 250-260).

6. La notion de non-lieu a été définie par Marc Augé dans son ouvrage Non-lieux (1992). À la différence du lieu qui est un espace qui nous convient, où l'on se sent "chez soi ", un non-lieu est, selon Marc Augé, un espace inhabitable : autoroute, gare, aéroport...

7. Dans un article récent, je me suis efforcé de montrer comment, après la revendication nostalgique d'une relocalisation paysagère de la ville de New York par la peinture impressionniste, un processus de dislocation avait permis la recomposition imaginaire, par le cinéma et la peinture, du paysage urbain de New York sur de nouvelles bases (Mottet, 2000).

8. J'emprunte cette expression à Thierry Jousse qui intitule l'un de ses articles sur "La Haine. Le Banlieue-film existe-t-il?" (1995).

9. Ma réflexion ici doit beaucoup à la démarche fondatrice de Michel de Certeau, développée dans son livre LInvention du quotidien. Arts de faire (1980, p. 179-236).

10. "Habiter une ville, nous dit Julien Gracq, c'est y tisser par ses allées et venues journalières, un lacis de parcours très généralement articulés autour de quelques axes directeurs" (1985, p. 2).

11. Dans sa théorie du "modulor", Le Corbusier proposait de composer certains éléments de l'habitat selon les postures fondamentales de l'homme quotidien (couché, debout, assis).

12. Le film de M. Kassovitz s'inscrit, ici aussi, dans une longue tradition de traitement de la banlieue par le cinéma français. Comme le note José Baldizzone: "Dans la plupart des cas la banlieue est liée à la ville proche" (Baldizzone, 1994, p. 109).

13. A l'opposé, un des événements significatifs des débuts du paysage, au XVI siècle, est précisément le développement concomitant de la notion de "paysage du monde". Des peintres comme Joachim Patinir ou Pierre Bruegel (dit l'ancien) montrent le monde dans la richesse de ses détails topographiques locaux : villages, fleuves, montagnes, forêts, cultures. Mais ces éléments de la terre se font alors "weltlandshaft», paysage du monde. Et la terre est envisagée comme espace universellement habitable. C'est seulement ensuite, suite à un processus historique d'individuation, que la perception du paysage s'établira sur le mode de la disjonction. Sur ce point voir notamment Georges Simmel (1998).

14. Les paysages de Bruegel, par exemple, représentent la terre comme espace à contempler, mais où l'on circule en tous sens. Il ne suffit pas de voir le monde, il faut le parcourir. Et le monde représenté est lui-même animé de mouvements possibles: chemins, rivières, horizon ouvert articulent parcours et paysage.

15. Sur ce point, on peut se reporter à mon analyse de la naissance de ce type d'espace au cinéma dans les courts métrages Biograph tournés par Griffith à New York (Mottet, 1998).

16. Sur ce point, voir L. Moinereau (1994).

17. On peut remarquer qu'en revanche une des caractéristiques principales de certaines banlieues pavillonnaires réside dans le silence, entouré par les bruits de la ville.

18. "Niquer" est le fruit d'une reverbalisation de "Quène", avec l'incorporation de sonorités arabes au verlan. Il y a là une défrancisation reflétant bien la situation des locuteurs qui, dans La Haine, parlent de la cité, le lieu d'où la langue tire son origine.

\section{RÉFÉRENCES BIBLIOGRAPHIQUES}

Arasse, 1996: Daniel Arasse, Le Détail, Paris, Flammarion, 1996.

Augé, 1992: Marc Augé, Non-lieux, Paris, Seuil, 1992.

Entre présent et possible: la difficile recomposition du paysage de la banlieue... 
Baldizzone, 1994 : José Baldizzone, "Carné, Blier et Rohmer vont... loin en Banlieue", Cabiers de la Cinémathèque, n" 59-60, Perpignan, institut Jean-Vigo, février 1994.

Bonnefoy, 1977 : Yves Bonnefoy, Le Nuage rouge : essais sur la poétique, Paris, Mercure de France, 1977.

Bordet, 1998 : Joëlle Bordet, Les Jeunes de la cité, Paris, PUF, 1998.

Carné, 1987: Marcel Carné, "Quand le cinéma descendra-t-il dans la rue?", dans Lise Grenier (dir.), Catalogue Cités-cinés, Paris, Ramsay, 1987.

Chion, 1995: Michel Chion, "L'homme qui marche", dans Jacques Aumont (dir.), L'Invention de la figure humaine, Conférences du Collège d'histoire de l'art cinématographique, 1995, p. 37-51.

Collot, 1988: Michel Collot, L'Horizon fabuleux, Paris, Corti, 1988.

Costa, 1999 : Antonio Costa, Cinema e pittura, Torino, Leosher Editore, 1999.

de Certeau, 1980 : Michel de Certeau, L'Invention du quotidien. Arts de faire, Paris, Union générale d'éditions, 1980.

Dubois, 1999 : Philippe Dubois, "Le regard vertical ou les transformations du paysage ", dans Mottet, 1999.

Goetz, 1997 : Benoît Goetz, "La dislocation : critique du lieu ", dans C. Younès et M. Mangematin (dir.), Lieux contemporains, Paris, Descartes et Cie, 1997.

Gracq, 1985 : Julien Gracq, La Forme d'une ville, Paris, José Corti, 1985.

Jousse, 1995: Thierry Jousse, "La Haine. Le Banlieue-film existe-t-il?", Cahiers du cinéma, n" 492, juin 1995.

Le Dantec, 1999 : Jean-Pierre Le Dantec, "Zones. Les paysages oubliés", dans Mottet, 1999 , p. $250-260$.

Lévinas, 1961 : Emmanuel Lévinas, Totalité et infini, Paris, Hachette, 1961.

Lynch, 1999: Kevin Lynch, L'Image de la cité, Paris, Dunod, [1960] 1999.

Lyotard, 1998 : Jean-François Lyotard, L'Inhumain, Paris, Galilée, 1998.

Mangematin et Younès, 1997 : Michel Mangematin et Chris Younès (dir.), Lieux contemporains, Paris, Descartes et Cie, 1997.

Moinereau, 1994: Laurence Moinereau, «Paysages de cinéma : les figures emblématiques d'une banlieue imaginaire ", Cahiers de la Cinémathèque, ns 59-60, février 1994, p. 35-39.

Molinier, 1999: Philippe Molinier, «Y a-t-il des paysages dans les films d'Éric Rohmer? ", dans Mottet, 1999.

Mottet, 1998 : Jean Mottet, L'Invention de la scène américaine, Paris, L'Harmattan, 1998.

Mottet, 1999 : Jean Mottet (dir.), Les Paysages du cinéma, Seyssel, Champ Vallon, 1999.

Mottet, 2001 : Jean Mottet, "Cinéma et peinture : la difficile invention du paysage de New York", in Leonardo Quaresima ex Laura Vichi (direcrion), La Decima Musa. Il cinema e le altre arti/The Tenth Muse. Cinema and other Arts, Udine/Gemona del Friuli, Forum, 2001, p. 255-261.

Roger, 1997 : Alain Roger, Court traité du paysage, Paris, Gallimard, 1997.

Rohmer, 1985 : Éric Rohmer, «Le Celluloïd et la pierre », entretien avec Éric Rohmer par Claude Beylie et Alain Carbonnier, Avant-Scène Cinéma, n 336, janvier 1985.

Sibony, 1998: Daniel Sibony, Violence, Paris, Seuil, 1998.

Simmel, 1998: Georges Simmel, "Philosophie du paysage", La Tragédie de la culture et autres essais, Paris/Marseille, Rivages, 1988.

Van Waerbeke, 1991 : Jacques Van Waerbeke, Images d'espaces de la banlieue de Paris, $X I X$ et XX siècles. Étude de géographie culturelle, Paris, Université de Paris XII, thèse dactylographiée, 2 volumes, 1991. 